\title{
ANÁLISE DE FATORES QUE INFLUENCIAM O PREÇO DA MADEIRA EM TORA PARA PROCESSAMENTO MECÂNICO NO PARANÁ
}

\author{
Alexandre Nascimento de Almeida ${ }^{1}$, João Carlos Garzel Leodoro da Silva ${ }^{2}$, \\ Humberto Ângelo ${ }^{3}$, Blas Enrique Caballero Nuñez ${ }^{4}$
}

(recebido: 5 de novembro de 2008; aceito: 31 de março de 2010)

\begin{abstract}
RESUMO: O entendimento da formação do preço é determinante para o planejamento das atividades de qualquer empresa, tornandose mais importante para o segmento florestal, visto o logo-prazo necessário para a produção de madeira. Neste trabalho, objetivouse estimar as elasticidades das principais variáveis que afetam o preço da madeira para o processamento mecânico no mercado paranaense. Para tanto, foi estimado um modelo econométrico que contou com as seguintes variáveis explicativas: a) renda per capita, b) taxa de câmbio, c) preço pago pelo mercado de celulose e d) produtividade. As estimativas foram realizadas por meio do método de mínimos quadrados ordinários (MQO) na forma funcional logarítmica. Os dados foram anuais e referem-se ao período de 1988 a 2004. Os resultados indicaram uma sensibilidade elástica à produtividade, próxima a unitária para a renda e inelástica ao câmbio e preço pago pelo mercado de celulose.
\end{abstract}

Palavras-chave: Mercado florestal, econometria, MQO.

\section{ANALYSIS OF FACTORS THAT INFLUENCE THE LOG PRICE FOR MECHANICAL PROCESS IN THE STATE OF PARANA}

\begin{abstract}
The understanding of price formulation is decisive to plan any activity and becomes more important to forests activities, since the production is take in long range. The objective of this work was to estimate the elasticity of the main variables that affect the stumpage price for the mechanical processing in the state of Parana's market. An econometric model was carried out with the following explanatory variables: per capita income, exchange rate, pulpwood price and productivity. The logarithmic functional form model was performed through ordinary least squares method (OLS). The annual data refers to 1988-2004 period. The results indicated an elastic sensibility to the productivity, close to the unitary for the per capita income and inelastic to exchange rate and pulpwood price.
\end{abstract}

Key words: Forest market, econometrics, OLS.

\section{INTRODUÇÃO}

O preço da madeira é um elemento-chave para o desenvolvimento de projetos florestais e o entendimento do seu comportamento é importante em razão da característica de longo prazo desse tipo de projeto.

$\mathrm{O}$ entendimento de como ocorre à formação do preço no mercado de madeira em tora no Paraná é complexo, pois se dá em um ambiente de concentração de mercado, tanto na oferta quanto na demanda de madeira. Além disso, é comum no Estado a interação entre o mercado de madeira mais fina para celulose e o de madeira mais grossa para o processamento mecânico.
Normalmente, as empresas ligadas ao processamento mecânico são de pequeno porte, de uso intensivo de mão-de-obra, poucas possuem reflorestamentos próprios e, portanto, são dependentes da madeira do mercado e altamente sensíveis às oscilações do seu preço.

O Paraná tem uma atuação de destaque no comércio de madeira plantada para o processamento mecânico, pois respondeu por aproximadamente $28 \%$ da produção nacional de madeira para a indústria de produtos sólidos em 2003. Contava ainda com quase 2.500 estabelecimentos responsáveis pelo desdobramento e fabricação de produtos de madeira, os quais empregam cerca de 45 mil pessoas (ABIMCI, 2006).

\footnotetext{
${ }^{1}$ Engenheiro Florestal, Doutorando em Ciências Florestais - Universidade Federal do Paraná/UFPR - Av. Lothário Meissner, 900 Jardim Botânico - Campus III - 80210-170 - Curitiba, PR - alexfloresta@ pop.com.br

${ }^{2}$ Engenheiro Florestal, Professor Doutor em Economia Aplicada - Departamento de Economia Rural e Extensão - Universidade Federal do Paraná/UFPR - Av. Lothário Meissner, 900 - Jardim Botânico - Campus III - 80210-170 - Curitiba, PR - garzel@ufpr.br

${ }^{3}$ Engenheiro Florestal, Professor Doutor em Economia e Política Florestal - Departamento de Engenharia Florestal - Faculdade de Tecnologia - Universidade de Brasília/UnB - Cx. P. 04357 - Asa Norte - 70919-970 - Brasília, DF - humb@unb.br

${ }^{4}$ Economista, Professor Doutor em Economia - Departamento de Economia - Universidade Federal do Paraná/UFPR - Av. Lothário Meissner, 632 - Jardim Botânico - 80210-170 - Curitiba, PR - blas@ufpr.br
} 
Durante a história florestal paranaense, o equilíbrio no mercado de madeira em tora para o processamento mecânico tem ocorrido à custa de bruscas variações no preço da madeira. Exemplo desse fato é que entre $1986 \mathrm{e}$ 1992 o preço real da madeira caiu $74 \%$ e, seis anos depois (período inferior a um ciclo de corte), entre 1999 e 2005 ele praticamente dobrou (INSTITUTO BRASILEIRO DE GEOGRAFIA E ESTATÍSTICA - IBGE, 2006; SECRETARIA DE AGRICULTURA E ABASTECIMENTO - SEAB, 2006). Esses dois períodos de grande variação do preço da madeira podem ser considerados, respectivamente, reflexos da entrada no mercado da madeira oriunda do ápice do período de incentivos fiscais e de um aumento da demanda externa por madeira (ALMEIDA, 2006).

No primeiro período (1986 a 1992), a demanda por madeira não acompanhou a sua oferta. O Brasil e o mundo viviam um período de baixo crescimento econômico e, em especial, o Brasil, se deparou com o insucesso dos planos Bresser e Collor para estabilização da economia, concomitante ao início da Guerra do Golfo e a Crise do Petróleo. Já, no segundo período (1999 a 2005), o aviltamento dos preços se deve muito à adoção de uma política de câmbio flutuante somado à desvalorização do real frente ao dólar, levando assim a uma forte pressão de demanda externa.

As variações no preço da madeira dificultam o planejamento da atividade visando a compatibilizar o consumo das indústrias de base florestal (demanda de madeira) e o aumento dos plantios florestais (oferta). Essa situação pode inibir investimentos no setor, principalmente de empresários de pequeno porte, visto que, normalmente, as grandes áreas de florestas estão ligadas às grandes indústrias.

Neste contexto, conduziu-se este trabalho, com o objetivo de estimar um modelo econométrico capaz de explicar o comportamento do preço da madeira para o processamento mecânico no mercado paranaense. Especificamente, objetivou-se identificar e estimar as elasticidades das principais variáveis que afetam o preço da tora para processamento mecânico no estado do Paraná, bem como avaliar o poder de previsão do modelo.

\section{METODOLOGIA}

\subsection{Referencial teórico}

Segundo Varian (1999), dificilmente, e só em pequenos espaços de tempo, as demandas e as ofertas das pessoas não sejam compatíveis. Este autor ainda afirma que isso até pode acontecer, mas normalmente não ocorre.
Em geral, os preços ajustam-se até que o total demandado pelas pessoas seja igual ao total ofertado. Klemperer (1996) comenta que o desequilíbrio no mercado de madeira nunca ocorre, pois a questão é em qual nível de preço a demanda será igual à oferta.

A formação do preço da madeira depende, principalmente, dos custos de produção, mas é o mercado quem determina ou estabelece esse preço, resultante do equilíbrio entre as forças de oferta e demanda. Assim, tanto as variáveis determinantes da oferta quanto da demanda de madeira devem ser consideradas na especificação de um modelo explicativo de preço.

Normalmente, as principais variáveis que afetam a demanda e a oferta de um produto são: preço, renda, preço de bens relacionados (substituto ou complementar), gostos e preferências, custos de produção, tecnologia e expectativas (MANKIW, 2005).

A demanda de madeira em tora depende da necessidade do consumidor final que, em geral, adquire a madeira sólida em duas formas principais: como móveis ou para uso na construção civil. Nesse último caso, podem ser produtos de maior valor agregado (PMVA), como portas, pisos, janelas, molduras, entre outros. Portanto, a demanda por madeira é dita derivada, ou seja, dependente da procura do consumidor final, sendo que esta por sua vez é chamada primária, uma vez que é onde tudo se inicia (ALMEIDA, 2006).

Dependendo do objetivo do trabalho podem-se utilizar variáveis referentes ao consumidor final ou a indústria responsável pelo processamento da madeira. Segundo Thompson (1993), quando a demanda por um bem ou serviço é derivada, pode-se obter um volume considerável de informação pela análise dos hábitos e das características dos usuários do produto final.

$\mathrm{Na}$ atividade florestal, o curto prazo não permite nenhum clico de produção e o longo prazo, onde todos os fatores são variáveis, somente é alcançado ao final de um ciclo de produção florestal. Desta forma, a escolha ótima dos fatores de produção a longo prazo no setor florestal é uma tarefa muito complexa e difícil de imaginar como factível (ALMEIDA, 2006). De acordo com Nautiyal \& Williams (1990), a dependência de um longo prazo ótimo, torna quase impossível a formulação de uma função de oferta de madeira a longo prazo sustentada pela teoria econômica clássica.

Apesar desta limitação, normalmente, as variáveis utilizadas para explicar a oferta de madeira em tora, além do preço, são:

Cerne, Lavras, v. 16, n. 2, p. 243-250, abr./jun. 2010 
- Inventário como uma proxy inversa para os custos de colheita. A justificativa é que com um aumento no volume de madeira em pé, os custos marginais de colheita percebidos em economias de escala diminuem (CARTER, 1992; DANIELS \& HYDE, 1985; HULTKRANTZ \& ARONSSON, 1989; NEWMAN, 1987). Naturalmente, esta proxy de custos apresenta limitações, além de ser uma variável que carece interpolação de dados, pois estes não são coletados anualmente.

- Ao invés da variável inventário é frequente a inclusão de uma variável autorregressiva de primeira ordem para oferta. Nesse caso, considera-se uma relação positiva entre a oferta no ano corrente e a quantidade de madeira negociada em anos prévios (ADAMS, 1975; HETEMÄKI \& KUULUVAINEN, 1992; LEUSCHNER, 1973; POLYAKOV et al., 2005; WIECHETECK, 2001). Aparentemente, a justificativa mais plausível para o uso dessa variável é captar o efeito das expectativas, porém, o suporte teórico dessa variável é muito frágil.

- Preço pago pelo mercado de celulose, objetivando explicar a concorrência ou a complementaridade de outros usos da madeira (BRÄNNLUND et al., 1985; NEWMAN, 1987).

Infelizmente, não existem séries históricas referentes à variável inventário para o Paraná. As séries mais próximas disponíveis são referentes à área de plantio das grandes empresas, porém, esses dados não são boas proxies para explicar a oferta de madeira, pois, dentre outras limitações, não discriminam áreas de florestas maduras das florestas jovens.

Ainda não existe estudo quantitativo definitivo referente à oferta de madeira em tora no mundo e, no Brasil, a situação ainda é mais precária. A dificuldade está principalmente nas fracas proposições teóricas existentes, na limitação de conhecimento referente à dinâmica do mercado nacional e nos grandes obstáculos para obtenção de dados.

\subsection{Referencial analítico}

O modelo econométrico explicativo do preço da madeira para o processamento mecânico no Paraná baseouse na seguinte equação:

$\ln P^{P M}=\alpha_{1}+\alpha_{2} \ln R I^{P C}+\alpha_{3} \ln C+\alpha_{4} \ln P^{C}+\alpha_{5} \ln P R O^{P}+\varepsilon$

$P^{P M}=$ preço da madeira para o processamento mecânico

$R I^{P C}=$ renda interna per capita

$C=$ taxa de câmbio efetiva real defasado em um período
$P^{C}=$ preço da madeira pago pelas indústrias de celulose $P R O^{P}=$ produtividade do Pinus medido pelo Incremento Médio Anual (IMA)

$\varepsilon=$ termo de erro da equação

O método utilizado na estimativa da Equação 1 foi o de Mínimos Quadrados Ordinários (MQO) e a forma funcional empregada foi a logarítmica, decorrente da possibilidade de obtenção direta das elasticidades.

Foi desconsiderada a influência de uma variável referente à "quantidade" de madeira produzida para o processamento mecânico na explicação do modelo. $\mathrm{O}$ motivo se deve à alta concentração do mercado de madeira no Estado.

Almeida (2006) afirma que aproximadamente 50\% dos plantios florestais no Paraná são de propriedade de poucas empresas, principalmente de papel e celulose sendo que apenas uma detém cerca de um quarto da área reflorestada do Estado, configurando-se como a maior fornecedora de toras para o processamento mecânico no Paraná (KLABIN, 2006).

Segundo Frank (1998), em uma estrutura concentrada pode não existir uma correspondência única entre o preço e quantidade o que dificulta encontrar uma relação significativa entre ambas as variáveis ao longo do tempo.

Para a renda per capita é esperado um efeito positivo no preço da madeira, pois normalmente um aumento da renda leva a um deslocamento da demanda para a direita e, consequentemente, a um aumento do preço.

O mercado externo é um grande consumidor de produtos sólidos de madeira brasileiros. Esse mercado consome aproximadamente $80 \%$ do compensado, $90 \%$, das molduras, $33 \%$ de painéis colados de madeira (EGP), $20 \%$ de móveis e $26 \%$ do piso de pinus produzidos no Brasil (SILVICONSULT, 2006). Dessa forma, é esperado que um aumento da taxa de câmbio (desvalorização da moeda nacional) leve a uma diminuição dos preços dos bens internos em termos de bens externos e, consequentemente, a um aumento da demanda externa e do preço da madeira no mercado interno.

A taxa de câmbio foi testada no presente trabalho em período corrente e defasada em um período. Normalmente, espera-se um efeito defasado do câmbio sobre o desempenho das exportações industriais. Segundo Scalco et al. (2008), no período de uma desvalorização cambial as quantidades de exportações e importações são usualmente pré-determinadas por contratos feitos em

Cerne, Lavras, v. 16, n. 2, p. 243-250, abr./jun. 2010 
períodos anteriores. Os volumes, portanto, responderão às mudanças na taxa de câmbio somente quando novos contratos são feitos, cujos efeitos são observados algum tempo depois da desvalorização/depreciação.

Conforme Brännlund et al. (1985) e Newman (1987), é esperada uma relação direta entre o preço pago pelo processamento mecânico e o preço pago pelo uso alternativo da madeira nas fábricas de celulose. Assim, um aumento do preço pago pela madeira pelo setor de celulose diminuiria a oferta de madeira para o segmento sólido e, consequentemente, acarretaria um aumento de seu preço.

Como uma proxy da evolução tecnológica, esperase um efeito indireto para produtividade. Um incremento na produtividade leva a um aumento da oferta de madeira e, consequentemente, a uma queda no seu preço.

Para o modelo estudado a constante não tem efeito prático, pois de acordo com Hair Junior et al. (2005) o teste $t$ raramente é necessário para os termos de intercepto. Os dados usados para desenvolver o modelo não incluem algumas observações em que todas as variáveis independentes assumam valor zero, o termo constante estaria "exterior" aos dados e atuaria apenas para posicionar o modelo. Nesse caso, não é necessário testar o termo constante (HAIR JUNIOR et al., 2005).

Outras hipóteses testadas no modelo são referentes à inexistência de problemas de multicolinearidade, heteroscedasticidade e autocorrelação. Essas hipóteses ajudam a estabelecer se as elasticidades estimadas têm propriedades desejáveis de não viés, eficiência e consistência.

Conforme Kmenta (1978) como a multicolinearidade é uma característica da amostra, não existe um "teste de multicolinearidade", mas pode-se medir o seu grau em qualquer amostra particular. A avaliação da multicolineariedade foi pelo indicador do Fator de Inflação da Variância (FIV) fornecido diretamente pelo SPSS. Como uma regra prática, Gujarati (2000) sugere que se o FIV de uma variável exceder a 10, diz-se que esta variável é altamente colinear.

A heteroscedasticidade foi avaliada por meio do teste BPG de Breusch \& Pagan (1979) e, a autocorrelaçao, pela tradicional estatística $d$ de Durbin-Watson.

Foram admitidos valores estatisticamente significativos aqueles com uma possibilidade de erro com até $5 \%$ de probabilidade. Todas as variáveis explicativas foram testadas por meio do teste $t$ unicaudal.

Por fim, foi avaliado o poder de previsão do modelo fora da amostra. O método utilizado foi de acordo com
Gujarati (2000), ou seja, foi re-estimado o modelo excluindo o grau de liberdade referente ao último período da amostra, no caso, o ano de 2004. A partir dos novos parâmetros estimados obteve-se um valor previsto para o ano de 2004, em seguida, comparou-se o valor previsto com o observado no mercado.

\subsection{Fonte de dados}

Os dados utilizados foram anuais e compreenderam o período de 1988 até 2004 e 1987 e 2004 para a taxa de câmbio.

A Renda per capita é medida por meio da razão entre a Renda Disponível Bruta e População, ambas as séries coletadas junto ao IBGE (2006). A Taxa de câmbio efetiva real é calculada pela média ponderada do índice de paridade do poder de compra dos 16 maiores parceiros comerciais do Brasil. Esse índice foi criado pelo IPEA (2006) visando a captar melhor o efeito do câmbio nas exportações.

O preço pago pela celulose foi obtido pela razão entre o valor e quantidade produzida de madeira em tora para celulose para o Paraná (IBGE, 2006) e corrigidos pelo Índice de Preços ao Consumidor Amplo - IPCA.

O preço da madeira pago pelo o processamento mecânico foi obtido da mesma forma e por meio da mesma fonte. Isso foi necessário, uma vez que inexiste uma série histórica governamental no Brasil em relação ao preço de celulose e processamento mecânico no Paraná para o mesmo período. Além disso, a série atende o objetivo neste trabalho e reflete melhor o comportamento do preço, comparativamente a uma coleta onde não são tomados os devidos cuidados com a amostragem.

A produtividade do Pinus foi obtida por meio da extrapolação de dados referentes a plantios nacionais (EMPRESA DE PESQUISA AGROPECUÁRIA E EXTENSÃO RURAL DE SANTA CATARINA - EPAGRI, 2005). Essa variável apresenta limitações, pois não se têm uma série periódica anual, somente constatações por meio de produtividades médias em períodos de anos (anos 70, anos 80 e pós 1995). Sabe-se que ganhos genéticos somente são verificados após pelo menos um ciclo e que a substituição de florestas antigas por plantios clonais é gradual e ocorre de maneira diferenciada nas diversas regiões do estado.

\section{RESULTADOS E DISCUSSÃO}

Os coeficientes estimados, tamanho da amostra, $\mathrm{R}^{2}$ aj e os resultados dos testes $t, \mathrm{~F}, d$ e valor do $P$ e FIV foram apresentados na Equação 2.

Cerne, Lavras, v. 16, n. 2, p. 243-250, abr./jun. 2010 


$$
\begin{aligned}
& \ln \hat{P}_{t}^{P M}=2,92+0,87 \ln R I_{t}^{P C}+0,43 \ln C_{t-1}+0,79 \ln P_{t}^{C}-1,6 \ln P R O_{t}^{P} \\
& \text { Teste } t=(1,67) \quad(3,93) \quad(1,74) \quad(5,47) \quad(-2,87) \\
& \text { Valor } P=(0,12) \quad(0,01) \quad(0,10) \quad(0,01) \quad(0,01) \\
& \text { FIV }=\quad(5,12) \quad(1,33) \quad(1,54) \quad(5,37) \\
& \mathrm{n}=17 \quad \mathrm{R}^{2} \mathrm{aj}=0,8 \quad \mathrm{~F}=17,04 \quad d=2,13
\end{aligned}
$$

Todas as variáveis apresentaram a direção de seus sinais conforme o esperado e, exceto para o câmbio, que foi estatisticamente significativa com uma possibilidade de erro de $5 \%$ de chance, todas as outras variáveis foram altamente significativas em um nível de significância inferior a $1 \%$.

Foi obtido um melhor ajuste da taxa de câmbio em um período defasado, sugerindo o efeito de contratos realizados em períodos anteriores. Porém, um melhor entendimento desse efeito vai além do objetivo, neste trabalho, ficando a sugestão para novos estudos que considerem séries com periodicidades mais curtas (mensais, trimestrais, semestrais).

O coeficiente de determinação ajustado $\left(R^{2} a j\right)$ indica que $80 \%$ da variação do preço foi explicada pelas variáveis pré-determinadas. O teste $\mathrm{F}$ foi altamente significativo e rejeitou a hipótese conjunta de que todos os coeficientes são simultaneamente iguais à zero com uma probabilidade de erro inferior a $1 \%$ de chance.

O indicador FIV sugeriu a ausência de multicolineariedade e os testes BPG e estatística $d$ rejeitaram a presença de heteroscedasticidade e autocorrelaçao em um nível de significância de $1 \%$.

A partir dos parâmetros da Equação 3, estimada com a exclusão da última observação da amostra, avaliouse o poder de previsão do modelo para o ano de 2004 (Figura 1).

$$
\begin{aligned}
& \ln \hat{P}_{t}^{\hat{P M}}=3,11+0,85 \ln R I_{t}^{P C}+0,39 \ln C_{t-1}+0,77 \ln P_{t}^{C}-1,58 \ln P R O_{t}{ }^{P} \\
& \text { Teste } t=(1,70) \quad(3,67) \quad(1,45) \quad(5,17) \\
& \text { Valor } P=(0,12) \quad(0,01) \quad(0,17) \quad(0,01) \quad(0,02) \\
& \mathrm{n}=16 \quad \mathrm{R}^{2} \mathrm{aj}=0,75 \quad \mathrm{~F}=12,38
\end{aligned}
$$

Conforme a Figura 1, o valor previsto para um período a frente da amostra apresentou um erro superior a $5 \%$, subestimando o valor observado em $12 \%$. Enquanto o valor previsto para o preço da madeira foi de $\mathrm{R} \$ 61,79$, o valor coletado no mercado foi de R \$ 70,00. Dentre alguns limitadores da eficiência do modelo para previsão destacase o pequeno tamanho da amostra e a obtenção de um $\mathrm{R}^{2}$ $(0,75)$ apenas razoável.

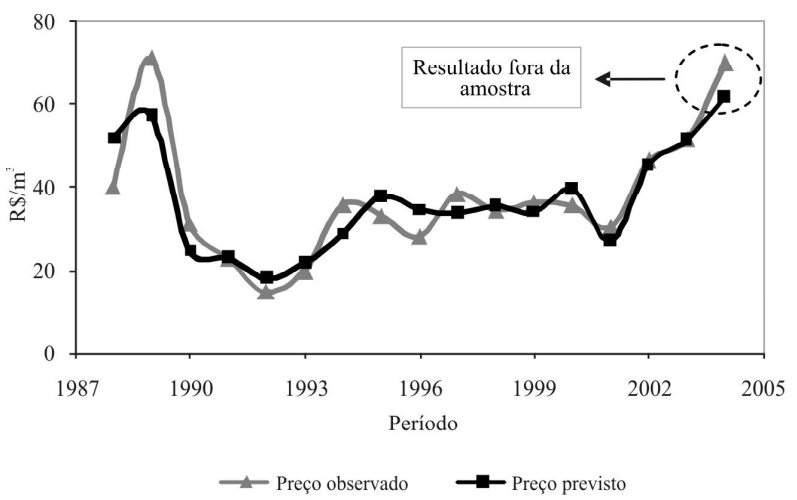

Fonte: IBGE (2006)

Figura 1 - Avaliação do poder de previsão do modelo.

Figure 1 - Evaluation of the model forecast power.

Comparativamente, os resultados das Equações 2 e 3 são semelhantes, porém, com a retirada de um grau de liberdade o câmbio passa a ser significativo apenas com um nível de significância de 8,5\%. Esses resultados refletem a sensibilidade dos resultados ao tamanho da amostra, contudo, perante a esta limitação pouco pode ser feito, pois os dados utilizados foram à melhor informação disponível no momento.

\subsection{Discussão dos Resultados}

A magnitude do efeito da renda per capita no preço da madeira para o processamento mecânico foi de que para cada $1 \%$ de aumento na renda per capita, tem-se um aumento de $0,87 \%$ no preço da madeira.

Os resultados sugerem uma considerável sensibilidade do preço da madeira a variações da renda, indicando que uma desaceleração da economia nacional terá um efeito próximo ao unitário na queda do preço da madeira para o processamento mecânico.

Esses resultados foram esperados visto que a indústria do processamento mecânico é uma das principais fornecedoras de matéria-prima para a indústria moveleira, as quais são sensíveis ao crescimento da economia nacional.

A elasticidade câmbio no preço da madeira para o processamento mecânico foi inelástica, pois para uma desvalorização de $1 \%$ da moeda nacional frente a seus principais parceiros comerciais espera-se um aumento no preço de apenas $0,43 \%$. Esses resultados indicaram que a adoção de políticas influentes na paridade cambial do Brasil não terá muito efeito no controle do preço da madeira.

Cerne, Lavras, v. 16, n. 2, p. 243-250, abr./jun. 2010 
Deve ser ressaltado que o entendimento de todos os resultados vale apenas para o período da amostra e considerando todas as demais condições (ceteris paribus). Qualquer extrapolação das mesmas deve ser feita com bastante cautela.

Especificamente, no caso do câmbio, deve-se verificar que a amostra contemplou dois períodos distintos, um entre 1987 a 1999 com uma política de câmbio fixo e outro após esta data, em que se adotou uma política de câmbio flutuante. Evidentemente, o efeito de uma oscilação do câmbio em uma política de câmbio fixo não tem a mesma resposta dos agentes de mercado caso a situação fosse de câmbio flutuante. Um modelo econométrico reflete o efeito histórico de uma variável e não o efeito momentâneo.

As relações entre os preços da madeira para celulose e processamento mecânico indicaram uma relação inelástica. $\mathrm{O}$ aumento de $1 \%$ no preço da primeira refletiu em um aumento de $0,76 \%$ no preço da segunda durante o período da amostra. A magnitude inelástica desse resultado, em parte, pode ser explicada pelo crescimento recente da indústria de painéis reconstituídos no Paraná com a implantação da Tafisa em 1998 e a partir de 2001 com a Masisa e Arauco. Essas empresas possivelmente contribuíram para uma maior concorrência e pressão no preço da madeira mais fina nas últimas duas décadas.

Outra questão que deve ser considerada é um provável excedente de madeira nas empresas de papel e celulose e, consequentemente, uma maior oferta de madeira grossa, haja vista o alto preço desta nos últimos anos, liberando assim áreas para novos plantios. Por fim, o entendimento desses resultados é limitado, já que depende do entendimento das estratégias das grandes empresas, visto o seu poder de mercado, tanto na oferta quanto na demanda de madeira.

No que tange a utilização de um provável poder de mercado das grandes empresas de base florestal a dúvida é se estrategicamente é interessante adotar esta política. Um pressuposto interesse das grandes empresas é tornar a atividade florestal atrativa para pequenos produtores e, para isso, faz-se necessário que o preço pago pela madeira possibilite uma receita superior a atividades agrícolas concorrentes.

Nos últimos anos, foi registrada uma ampliação dos programas de fomento das grandes empresas, o que tem resultado em um crescimento da participação de pequenos produtores na atividade florestal no Estado. Deve ser acrescentado que, muito mais importante do que o preço da madeira para o setor de papel e celulose é a garantia de suprimento da mesma. A produção de celulose requer altos custos fixos na instalação de suas fábricas e, consequentemente, os seus administradores devem assegurar primeiramente que estas operem continuamente para posteriormente advir o lucro com o comércio de madeira. Dois foram os trabalhos que indicaram a baixa importância do preço da madeira na sua demanda pelas empresas de papel e celulose (LEUSCHNER, 1973; POLYAKOV et al., 2005).

Em relação à estrutura do mercado de madeira em tora os resultados do modelo sugerem um poder de mercado das grandes empresas, visto a independência da variável "quantidade" para o ajuste do modelo. Porém, a independência da variável quantidade não quer dizer que exista um controle agressivo de preços pelas grandes empresas e, tampouco, que as mesmas tenham um poder excessivo sobre o preço. Se assim o fosse, seria impossível explicar o preço da madeira com variáveis do mercado e exógenas ao controle das grandes empresas, como a renda e taxa de câmbio. Além disso, os resultados sugeriram que os ganhos de produtividade foram repassados no preço da madeira e uma concorrência entre os mercados de celulose e processamento mecânico.

Os resultados para o efeito da produtividade foram elásticos, indicando que o aumento de $1 \%$ da produtividade leva a uma queda de $1,6 \%$ no preço da madeira para o processamento mecânico. Entretanto, a magnitude desses resultados deve ser analisada com cautela tendo em vista a evidente interpolação de dados que a série apresenta. Em geral, os resultados sugeriram uma forte influência da produtividade no preço da madeira.

O valor previsto pelo modelo fora do período da amostra não encoraja a utilização do mesmo para fins de previsão. Esses resultados foram esperados, pois normalmente os modelos estimados por mínimos quadrados ordinários não são utilizados para este fim. Segundo Gujarati (2000), os modelos baseados em mínimos quadrados ordinários dominaram a previsão econômica durante as décadas de 60 e 70, mas nos últimos tempos o glamour de tais previsões tem diminuído graças aos choques do petróleo de 1973 e 1979 e à crítica de Lucas (1976). O ataque dessa crítica está no fato de que os parâmetros estimados não são invariáveis na presença de mudanças na política, ou seja, não é possível garantir que as elasticidades no futuro serão as mesmas.

Entretanto, apesar do modelo ter se mostrado limitado para a realização de previsões, as elasticidades obtidas não são invalidadas. A avaliação da previsão é um

Cerne, Lavras, v. 16, n. 2, p. 243-250, abr./jun. 2010 
teste independente dos testes estatísticos e econométricos aplicados anteriormente. De acordo com Stock \& Watson (2004) não é necessário uma relação causal para fazer previsões e ignorar as interpretações causais libera a busca por boas previsões. Contudo, em algumas aplicações, a tarefa não é desenvolver um modelo de previsão, mas sim estimar relações causais entre variáveis de séries temporais.

\section{CONCLUSÕES}

As análises dos dados, por meio da metodologia utilizada indicam:

- sensibilidade próxima a unitária do preço da madeira para processamento mecânico diante as oscilações da renda per capita;

- influência inelástica da taxa de câmbio efetiva;

- efeito inelástico do preço da madeira para celulose no preço pago pelo processamento mecânico; madeira;

- forte influência da produtividade no preço da

- inadequação para a realização de previsões.

Sugere-se a utilização dos modelos VAR e ARIMA ou outras abordagens metodológicas por meio da visão prospectiva e metodologia de cenários para obtenção de previsões mais acuradas.

\section{REFERÊNCIAS BIBLIOGRÁFICAS}

ABIMCI. Setor de processamento mecânico da madeira no

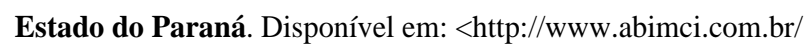
iport/06̄Docs/06̄ uadroDocs.htmì' Acesso em: 2 ago. 2006.

ADAMS, D. M. A model of pulpwood production and trade in Wisconsin and the Lake States. Forest Science, Chicago, v. 21, n. 3, p. 301-312, 1975.

ALMEIDA, A. N. Estudo econométrico da demanda e oferta de madeira em tora para o processamento mecânico no estado do Paraná. 2006. 217 f. Dissertação (Mestrado em Ciências Florestais) - Universidade Federal do Paraná, Curitiba, 2006.

BRÄNNLUND, R.; JOHANSSON, P. O.; LOFGREN, K. G. An econometric analysis of aggregate sawtimber and pulpwood supply in Sweden. Forest Science, Chicago, v. 31, n. 3, p. 595606, 1985.

BREUSCH, T.; PAGAN, A. A simple test for heteroscedasticity and random coefficient variation. Econometrica, v. 47, p. 12871294, 1979 .
CARTER, D. R. Effects of supply and demand determinants on pulpwood stumpage quantity and price in Texas. Forest Science, Chicago, v. 38, n. 3, p. 652-660, 1992.

DANIELS, B. J.; HYDE, W. F. Estimation of supply and demand for North Carolina's timber. Forest Ecology and Management, Amsterdam, v. 14, p. 59-67, 1986.

EMPRESA DE PESQUISA AGROPECUÁRIA E EXTENSÃO RURAL DE SANTA CATARINA. Centro de Estudos de Safra e Mercado. O setor florestal de Santa Catarina. Florianópolis, 2005. CD-ROM.

FRANK, R. H. Microeconomia e comportamento. 3. ed. Lisboa: McGraw Hill, 1998. 745 p.

HAIR JUNIOR, J. F.; ANDERSON, R. E.; TATHAM, R. L.; BLACK, W. C. Análise multivariada de dados. 5. ed. Porto Alegre: Bookman, 2005. 593 p.

HETEMÄKI, L.; KUULUVAINEN, J. Incorporating data and theory in roundwood supply and demand estimation. American Journal of Agricultural Economics, v. 74, n. 4, p. 1010-1018, 1992.

HULTKRANTZ, L.; ARONSSON, T. Factors affecting the supply and demand of timber from private nonindustrial lands in Sweden: an econometric study. Forest Science, Chicago, v. 35, n. 4, p. 946-961, 1989.

INSTITUTO BRASILEIRO DE GEOGRAFIA E ESTATÍSTICA. Anuário estatístico brasileiro. Rio de Janeiro, 2006.

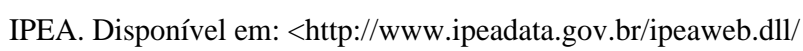
ipeadata?13 57421>. Acesso em: 2 dez. 2006.

KLABIN. Disponível em: Kww.klabin.com.br/pt-r/produtos/, icategoria.aspx?id=18y. Acesso em: 13 dez. 2006.

KLEMPERER, W. D. Forest resource economics and finance. New York: McGraw-Hill, 1996. 551 p.

KMENTA, J. Elementos de econometria. New York: Macmillan, 1978. $670 \mathrm{p}$.

LEUSCHNER, W. A. An econometric analysis of the Wisconsin Aspen pulpwood market. Forest Science, Chicago, v. 19, n. 1, p. 41-46, 1973.

Cerne, Lavras, v. 16, n. 2, p. 243-250, abr./jun. 2010 
LUCAS, R. E. Econometric policy evaluation: a critique. Carnegie-Rochesteer Conference Series, The Phillips Curve, Amsterdam, p. 19-46, 1976.

MANKIW, N. G. Introdução à economia-princípios de micro e macroeconomia. 2. ed. Rio de Janeiro: Elsevier, 2001. 831 p.

NAUTIYAL, J. C.; WILLIAMS, J. S. The long-run timber supply function. Forest Science, Chicago, v. 36, n. 1, p. 77-86, 1990.

NEWMAN, D. H. An econometric analysis of the southern softwood stumpage market: 1950-1980. Forest Science, Chicago, v. 33, n. 4, p. 932-945, 1987.

POLYAKOV, M.; TEETER, L. D.; JACKSON, J. D. Econometric analysis of Alabama's pulpwood market. Forest Products Journal, Amsterdam, v. 55, n. 1, p. 41-44, 2005.

SCALCO, P. R.; CARVALHO, H. D.; CAMPOS, A. C. Efeitos de curto e longo prazos de choques na taxa de câmbio real sobre o saldo da balança comercial agropecuária brasileira. In: CONGRESSO DA SOCIEDADE BRASILEIRA DE ECONOMIA, ADMINISTRAÇÃO E SOCIOLOGIA RURAL,

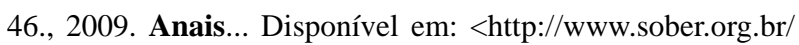
palestra /9/620.pdf>. Acesso em: 21 maio 2009.

SECRETARIA DE AGRICULTURA E ABASTECIMENTO. Relatório de atividades 2006. Curitiba, 2006.

SILVICONSULT. Evolução do mercado de pinus. Disponível em: swww.porthuseventos.com.br/congressopinus/>i Acesso em: 15 dez. 2006.

STOCK, J. H.; WATSON, M. W. Econometria. São Paulo: A. Wesley, 2004. 485 p.

THOMPSON, A. A.; FORMBY, J. P. Microeconomia da firma: teoria e prática. 6. ed. Rio de Janeiro: Prentice-Hall do Brasil, 1998. 359 p.

VARIAN, H. R. Microeconomia: princípios básicos. 4. ed. Rio de Janeiro: Campus, 1999.

WIECHETECK, M. S. S. Spatial equilibrium analysis of conifer timber markets in southern brazil and in the other mercosur countries. 2001. 237 f. Thesis (Ph.D.) - Michigan State, Michigan, 2001. 